\title{
Three-dimensional culture model to distinguish normal from malignant human bronchial epithelial cells
}

\author{
Delphine Fessart ${ }^{1,2}$, Hugues Begueret ${ }^{3}$ and Frederic Delom ${ }^{1,2}$
}

Affiliations: 'Université Bordeaux, Centre de Recherche Cardio-Thoracique de Bordeaux, U1045, Bordeaux, ${ }^{2}$ INSERM, Centre de Recherche Cardio-Thoracique de Bordeaux, U1045, Bordeaux, and ${ }^{3}$ Dept of Pathology, University Hospital of Bordeaux, Hôpital Haut-Lévêque, Pessac, France.

Correspondence: D. Fessart, Bordeaux University, Bordeaux Cardiothoracic Research Center, 146, Rue LéoSaignat, 33076 Bordeaux, France. E-mail: delphine.fessart@yahoo.fr

ABSTRACT In the present study, we have developed an in vitro three-dimensional model to differentiate normal lung cells from lung cancer cells in order to study the mechanisms resulting in lung cancer.

Using a reconstituted laminin-rich basement membrane (Matrigel), we were able to culture normal human bronchial epithelial cells and a subset of malignant cells. The two cell types can be readily distinguished by the ability of normal cells to express a structurally and functionally differentiated phenotype within Matrigel. Human bronchial epithelial cells embedded in Matrigel as single cells were able to form multi-cellular spherical colonies with a final size close to that of true acini in situ. Sections of mature spheres revealed a central lumen surrounded by polarised epithelial cells. In contrast, none of malignant cells tested, cell lines and lung biopsies responded to basement membrane by lumen formation.

These results demonstrated that this in vitro glandular tumour model can be useful for studies of bronchial oncogene. Indeed, these findings may provide the basis for a rapid assay to discriminate normal human bronchial epithelial cells from their malignant counterparts.

In conclusion, the three-dimensional tumour bronchial epithelial acinar-like sphere represents a novel in vitro model to further investigate pathophysiological functions resulting in lung cancer.

@ERSpublications

3D tumour bronchial epithelial acinar-like sphere is novel in vitro model to investigate changes resulting in lung cancer http://ow.ly/o8eDu

This article has supplementary material available from www.erj.ersjournals.com

Received: July 302012 | Accepted after revision: Dec 222012 | First published online: Jan 242013

Support statement: This work was supported by grants from Chaire d'Excellence INSERM-Universite de Bordeaux (grant number CRE08PREST) and Comité Aquitaine Charentes de la Ligue Nationale Contre le Cancer (grant number 2012-0038).

Conflict of interest: None declared.

Copyright @ERS 2013 


\section{Introduction}

Lung cancer is the most common cause of cancer-related mortality worldwide. Most human cancers arise from epithelial cells and tissues. Of lung cancers diagnosed, $>80 \%$ are nonsmall cell lung cancer (NSCLC), which includes adenocarcinoma, squamous cell carcinoma and large cell carcinoma [1]. In the past decades, although many tumour suppressor genes and oncogenes have been identified, the majority of NSCLC patients are usually diagnosed during the later stages of lung cancer and die without successful treatment [1-3]. The most common histological subtype of lung cancer is adenocarcinoma, accounting for almost half of all lung cancers in most countries $[4,5]$.

In order to translate the findings from basic cellular research into clinical applications, cell-based models need to recapitulate both the three-dimensional organisation and multicellular complexity of an organ, but at the same time need to accommodate systematic experimental intervention. Extracellular matrix is a key regulator of normal homeostasis and tissue phenotype [6]. Important signals are lost when cells are cultured in vitro on two-dimensional plastic substrata. Many of these crucial micro-environmental cues may be restored using three-dimensional cultures [7]. It has have been shown that signal transduction pathways in nonmalignant cells are integrated in three-dimensional cultures in ways not observed when cells are cultured as monolayer. For example, the expression and activity of $\beta 1$-integrin and epidermal growth factor receptor are reciprocally downregulated in breast cancer cells treated with various signalling inhibitors, but only when cultured on three-dimensional substrata [8]. However, efforts to study early events in human bronchial tumour development have been hampered by the lack of adequate means to distinguish between normal and transformed cells in culture. Epithelial tissues have several distinguishing microscopic features, such as a polarised morphology, specialised cell-cell contacts and attachment to an underlying basement membrane. Most internal epithelial organs consist of a monolayer of polarised cells lining a central cavity [9], which can be spherical, forming acini, or like a tube, forming a branching tubular tree. All of these features are necessary for the proper control of cellular proliferation, survival, differentiation and secretion [10]. This intact, well-ordered architecture is disrupted during the pathogenesis of epithelial tumours. Previous studies from primary human tumour tissue and animal models of lung epithelial tumours have provided information on the genetic events involved in lung cancer formation. Although these approaches have been critical for understanding lung epithelial tumours, they are relatively intractable for studying the biochemical and cell biological pathways involved in tumour formation, especially the mechanisms responsible for early oncogenesis. So far, the molecular signalling machineries involved in oncogenic transformation have been essentially studied on two-dimensional monolayers. However, because twodimensional monolayer cells do not recapitulate the glandular structure of epithelium in vivo, they do not provide the optimal system for fully understanding the regulation of proliferation, cell death and differentiation both in normal cell and also during early tumour formation. Thus, the mechanisms orchestrating the formation from individual cells into a well-ordered polarised glandular architecture remain unanswered by the system of a two-dimensional cellular monolayer culture. Therefore, we have used a three-dimensional culture model of normal and cancer human bronchial epithelial cells (HBECs). Different studies have used a reconstituted Matrigel (BD Biosciences, Franklin Lakes, NJ, USA) derived from the Englebreth-Holm-Swarm tumour, composed of laminin, collagen IV and entactin, to study the morphology of human epithelial cell in three-dimensional culture from breast [11], intestine [12, 13], pancreas [14, 15] and metastatic tumour cells [16]. Lung epithelial cells from distal regions of rodent airways have also been shown to grow in Matrigel [17, 18] and more recently in normal HBECs [19]. In their study, Wu et al. [19] have shown that normal human bronchial epithelial cells could differentiate into three-dimensional spheroids when grown on Matrigel. FrAnzDótTir et al. [20] have also shown that human bronchial epithelial cell line could be induced by endothelial cells to form branching bronchioalveolar-like structure in three-dimensional culture. A previous study on lung cancer using a slightly different threedimensional model, the collagen gel model [21], has also been reported. However, it has been shown by others [21] that, in collagen, both normal and tumour cells formed only loose aggregates, with poor or no cell-cell contacts, often displaying a fibroblast-like growth pattern. In contrast, Matrigel strongly supports both growth and differentiation of normal and spheroids. The three-dimensional organotypic culture systems, in Matrigel, have already been used as powerful cell-based model to investigate the functions of cancer genes and pathways in a biologically relevant context and high-throughput manner [10, 22], but oddly never in the lung.

We describe herein the growth of cancer HBECs in three-dimensional basement membrane culture with Matrigel. By comparison between three-dimensional architecture in normal and malignant cell lines, we were able distinguish normal and malignant human bronchial cells. This system could also be used to define differentiation markers and to delineate early changes in transformation assays or pre-neoplastic lesions. Thus, our three-dimensional HBEC culture provides the appropriate structural and functional context fundamental 
for examining biological activities in lung cancer and also to discover the mechanisms and signalling pathways responsible for organising individual cells into a well-ordered polarised glandular architecture.

\section{Materials and methods}

For expanded and detailed materials and methods please refer to the online supplementary material.

\section{Antibodies and reagents}

We used commercial matrix (Matrigel) for the organotypic assays. The sources of the primary antibodies used in these studies were as follows: $\beta$-catenin (BD Biosciences), p63 (clone 4A4; Santa Cruz Biotechnology, Santa Cruz, CA, USA), cleaved-caspase 3 (Asp175) (Cell Signaling, Danvers, MA, USA), mucin (MUC)5B (Sigma-Aldrich, St Louis, MO, USA), lysozyme (AZGP1; Sigma-Aldrich) and $\beta$-tubulin IV (clone ONS.1A6) (Sigma-Aldrich). Each primary antibody was used at 1:250 dilution factor and the secondary at 1:500 dilution factor. The secondary antibodies used were as follows: Alexa-Fluor-488- and Alexa-Fluor-546-conjugated anti-mouse and rabbit IgGs (Molecular Probes/Invitrogen, Grand island, NY, USA). Reagents used in the study were diaminophenylindole (DAPI) (Sigma-Aldrich) and paraformaldehyde (Sigma-Aldrich).

\section{Results}

\section{Organotypic culture of normal HBECs and carcinoma cells in basement matrix}

We first defined the behaviour of normal HBECs in two different conditions: on tissue culture plastic for two-dimensional culture (fig. 1a, left panel) and on Matrigel for three-dimensional culture (fig. 1b, left panels). HBECs on two-dimensional monolayer cultures grew exponentially (fig. 1a), as previously described [19]. Cells were then embedded in Matrigel matrix as single cells (fig. 1b and c, left panels). Within 10 days of culture, cells within the matrix had formed a population of almost uniformly spherical colonies (fig. 1c, left panels). Resemblance to acini in vivo was further confirmed by the appearance of small
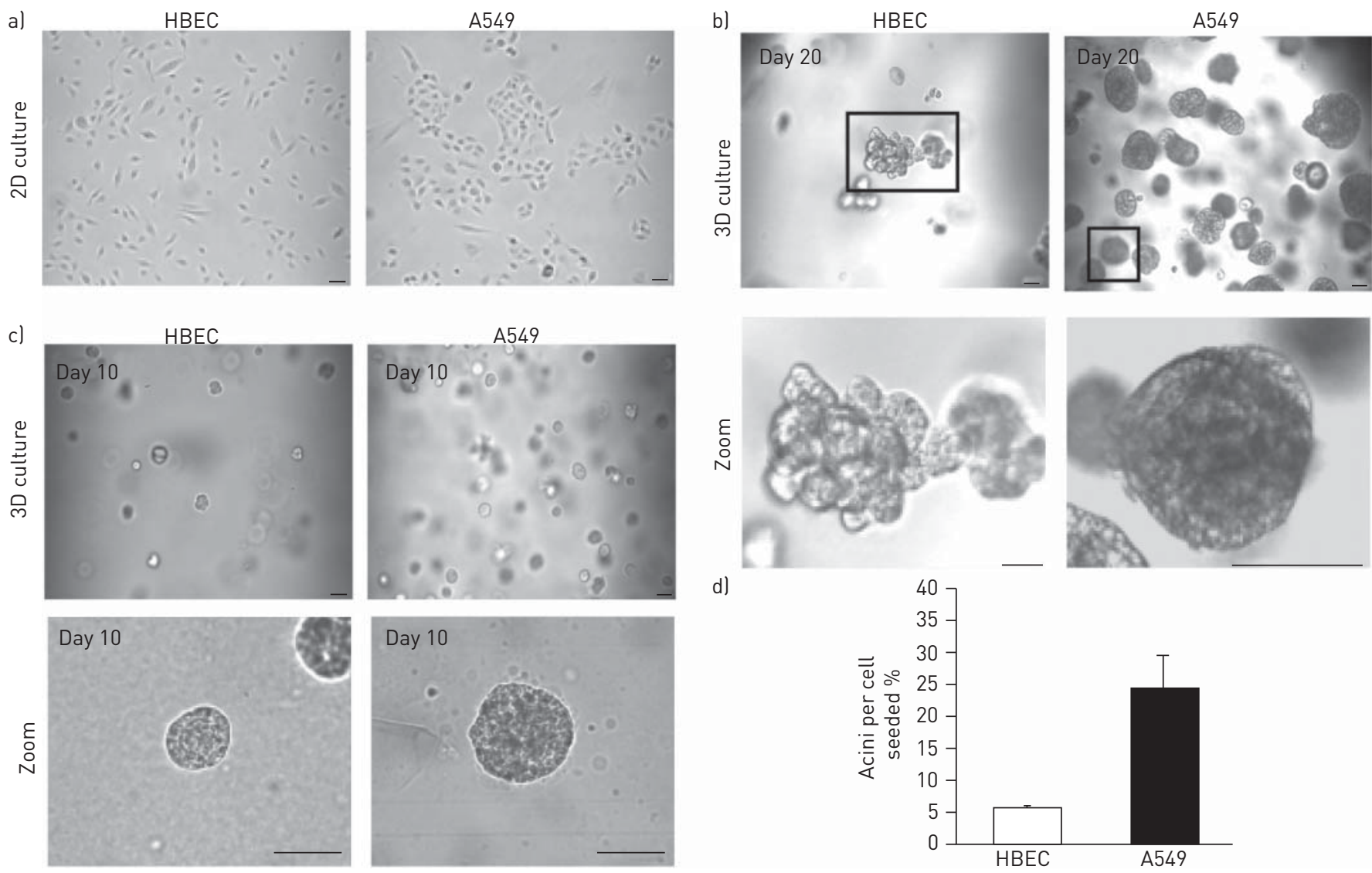

FIGURE 1 Organotypic culture of normal and malignant human bronchial epithelial cells (HBECs). Phase contrast of HBEC grown in two different culture conditions two-dimensional (2D) (a) and three-dimensional culture (3D) (b) as compared with A549 cells grown in the same conditions at day 20. c) Representative pictures of the 3D field after 10 days in culture. d) Quantification of the percentage of acini formed per cell seeded for HBECs and A549 cells after 10 days of $3 \mathrm{D}$ culture. Scale bars $=50 \mu \mathrm{m}$. 
connecting ducts between the spheres (fig. 1b, left panels). In contrast, cells grown in a two-dimensional monolayer did not form spheres and grew for few passages before cell senescence (fig. 1a, data not shown). As the most common histological subtype of lung cancer is adenocarcinoma, we analysed the pattern of adenocarcinoma (A549) cell growth under similar conditions. A549 cells grow in both conditions, on twodimensional monolayer cultures (fig. 1a, right panel) and within the matrix (fig. $1 \mathrm{~b}$ and c, right panels). However, in contrast to HBECs, which differentiate in three-dimensional culture at a late time-point (fig. 1b, left panels), A549 remains in an acini shape (fig. 1b, right panels). These data demonstrated that HBECs are capable of undergoing differentiation to form structures with markedly different morphology compared with A549 carcinoma cells (fig. 1b). Moreover, the A549 cell line in the matrix formed spheres with significantly higher acini number as compared with HBECs (fig. 1c).

\section{Differentiation of normal and carcinoma cells in the matrix}

The behaviour of normal cells (HBEC) and cancer cells (A549) in the matrix was further determined by markers that have been extensively used to describe tissue (acinar) polarity, such as baso-lateral expression of the cell-cell junction marker, $\beta$-catenin. Confocal analysis demonstrated that HBEC acini have developed cell-cell junction complexes (fig. 2a, upper panels), whereas A549 acini have disrupted cell-cell junctions (fig. 2a, lower panels). Basal cells of the human airway epithelium express the nuclear protein p63, which is not expressed by other airway epithelial cell [20]. HBEC acini showed a polarised nuclear apical expression of p63 (fig. 2b, upper panels). In contrast, A549 acini did not express this marker in the nuclei (fig. 2b, lower panels). Specificity of the Alexa-488 antibody was verified in figure $2 \mathrm{c}$. In parallel, we explored the localisation and the expression of these two markers in two-dimensional monolayer (online supplementary fig. S1). HBEC and A549 expressed $\beta$-catenin, but the cell junction marker localisation was different between HBEC and A549 cells (online supplementary fig. S1a). The membrane staining of $\beta$-catenin was more pronounced in A549 cells, as compared with HBEC cells, for which only few cells express a membrane localisation of $\beta$-catenin (online supplementary fig. S1a). The basal cell marker (p63) was expressed in HBEC with different expression intensity (online supplementary fig. S1b, upper panels), and was almost entirely absent in A549, with the exception of very few cells (online supplementary fig. S1b, lower panels).

\section{Acini secretion similar to respiratory tract glands}

To further characterise the differentiated acinar cells and compared with in vivo glandular acini in respiratory tract we compared the expression of MUC5B (a sumucosal glandular cell marker) (fig. 3) [23], AZGP1 (a glandular serous cell marker) and $\beta$-tubulin IV (a ciliated cell marker) [24]. As described by WU et al. [19], in HBECs, MUC5B and AZGP1 were distributed generally in luminal cells suspended within the lumen of the spheres and some were localised at the apical membrane (fig. $3 \mathrm{a}$ and b, upper panels). A549 also expressed the submucosal glandular cell marker, MUC5B (fig. 3a, lower panels). In contrast to HBEC, the glandular serous cell marker, AZGP1, was not detectable in A549 cell line (fig. 3b, lower panels). As previously reported [19], $\beta$-tubulin IV was not detectable in HBEC differentiated acini (fig. 3c, upper panels). In contrast, this ciliated cell marker was expressed at the basal surface of A549 cells in contact with the matrix (fig. 3c, lower panels). This staining phenotype at the edge of the sphere has been already reported in bronchial cells for cytokeratin 14 and $\beta 4$-integrin by FRANZDÓTTIR et al. [20]. Specificity of the Alexa-546 antibody was verified in figure $3 \mathrm{~d}$. In two-dimensional monolayer culture, in the same manner as in the three-dimensional matrix culture, MUC5B (online supplementary fig. S2a) was expressed in both cell lines. In contrast to three-dimensional culture, AZGP1 (online supplementary fig. S2a) and $\beta$-tubulin IV (fig. S2a) markers were expressed in both cell lines in two-dimensional monolayer culture.

\section{Distinctive growth regulation of $A 549$ versus $H B E C$ in matrix}

In the early stages of culture, cells form clusters surrounded by the matrix. As cultures progress, two distinct cell populations emerge within each acinus: polarised cells around the outer layer in contact with the matrix and nonpolarised cells comprising the inner region. With further culturing, these inner cells undergo cell death and clearance, leading to lumen formation. HBEC cells organise into cyst-like structures that contain a spherical layer of cells that enclose a central lumen (fig. 4a). Apoptosis was implicated in the process of cavitation or lumen formation, in a solid cell mass in several three-dimensional spheroid models [25-28]. A hallmark of most glandular epithelial tumours (breast, prostate, intestine, etc.) is the absence of a hollow lumen. Many types of breast cancer lesions, such as ductal carcinoma in situ, are characterised by loss of acinar organisation and filling of the luminal space [29]. However, it is not known if we can extend these observations to lung cancer cells. To assess the development of lumen in HBECs as compared with A549 cells, confocal z-stack imaging of acini using DAPI as nuclear marker was monitored at $20-\mu \mathrm{m}$ sections through the acini (fig. 4). In HBECs, two populations of cells within each acinus were observed: an outer layer in contact with the matrix and an inner subset that lacked contact with the matrix (fig. 4a). We also observed that HBECs could be easily distinguished from A549 cells by the fact that A549 cells filled the 

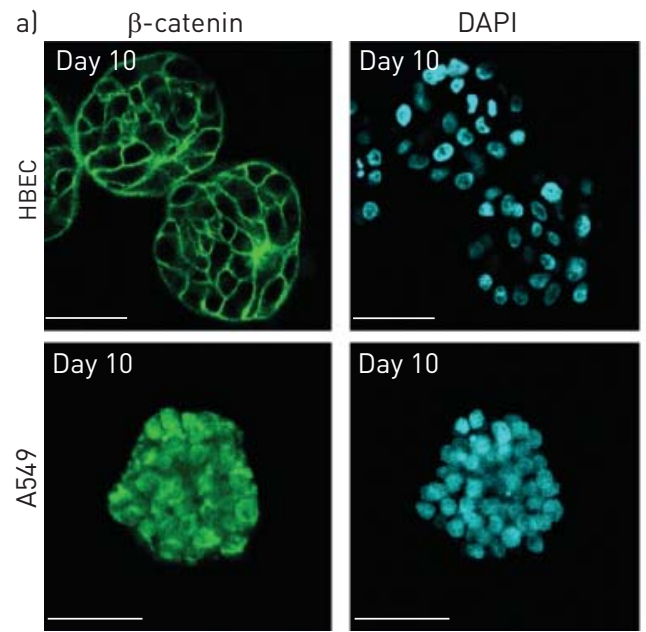

b)

p63
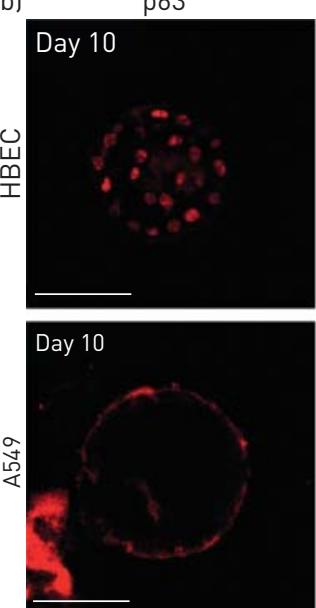

c) Anti-mouse-488

FIGURE 2 Immunofluorescence of acini generated by human bronchial epithelial cells (HBECs) as compared with cancer (A549) cell lines. Images are confocal cross-sections through the acin immunostained with a) $\beta$-catenin cell junction marker (green) and b) p63 basal cell marker (red); c) nuclei were counterstained with diaminophenylindole (DAPI).

c) Negative control sections were stained with secondary antibody alone (Alexa-488). Images are representative of the majority of acini at day 10 in culture. Scale bars $=50 \mu \mathrm{m}$. Images were acquired by confocal microscopy Nikon Eclipse 2000-C1 (Nikon, Tokyo, Japan).
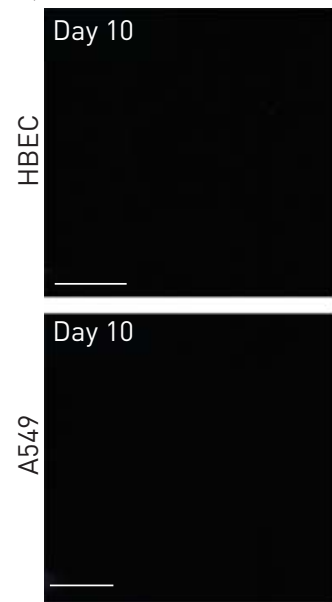

DAPI

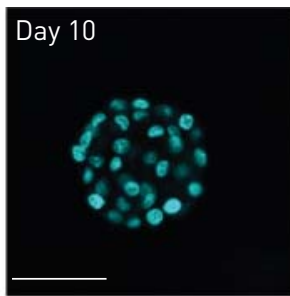

\section{Day 10}

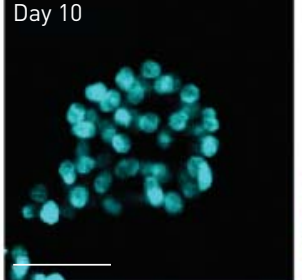

DAPI
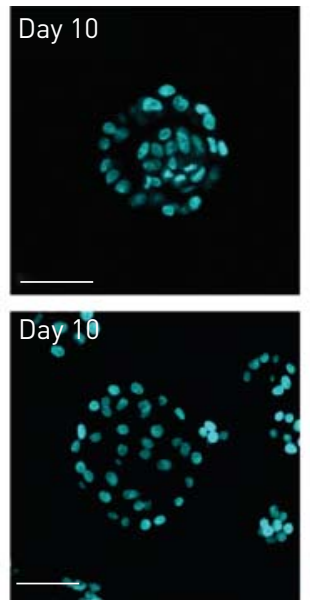

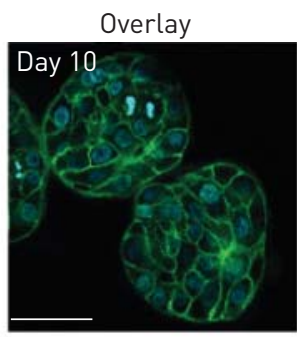

Day 10

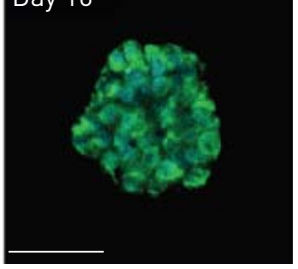

Overlay

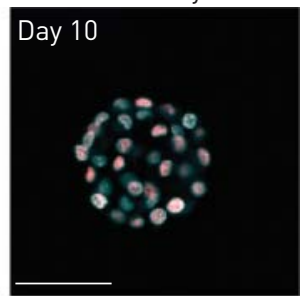

Day 10

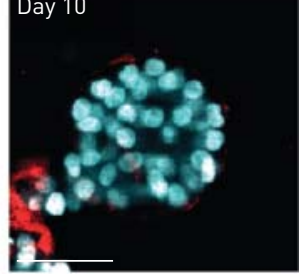

Overlay
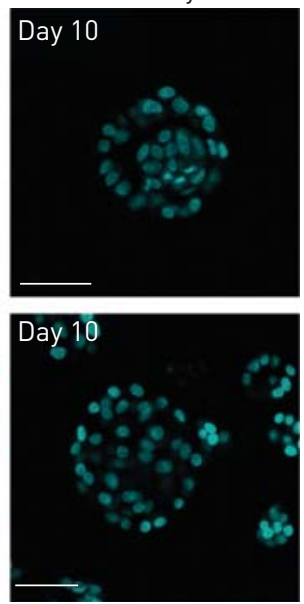

lumen (fig. 4b) whereas HBECs enclosed the lumen (fig 4a). In several epithelial cell types, apoptosis contributes to lumen formation; indeed, apoptotic cells are present in the developing lumen of salivary gland [27] and Madin-Darby canine kidney cysts [30], as well as mammary spheroids [25, 28, 31]. As previously reported by Wu et al. [19], we have grown cells in three-dimensional culture for 13 days to be able to detect caspase-3 activation. HBECs undergoing apoptosis in the luminal space was measured by cleaved activated caspase-3 staining (fig. 5, upper and middle panels). In contrast, A549 cells did not form a hollow lumen (fig. 4b) and are negative for caspase-3 staining (fig. 5, lower panels). These results 
a)
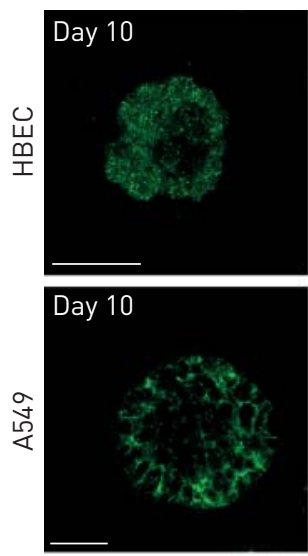

c)
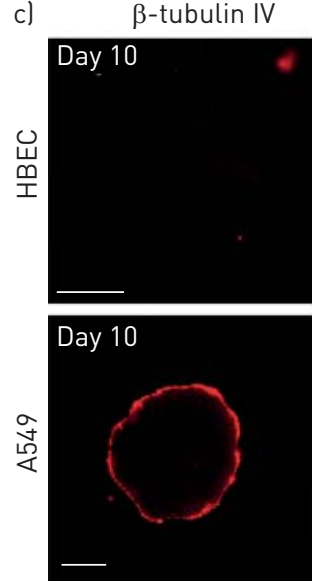

DAPI
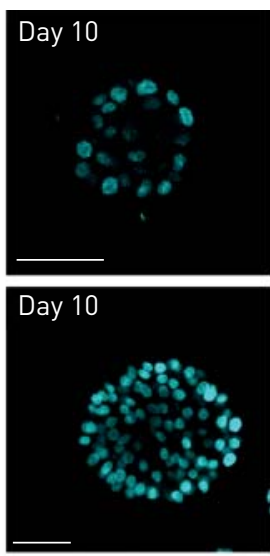

DAPI
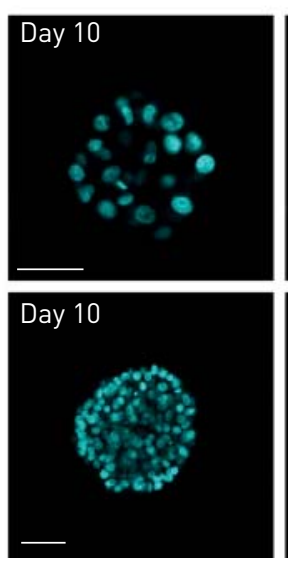

Overlay
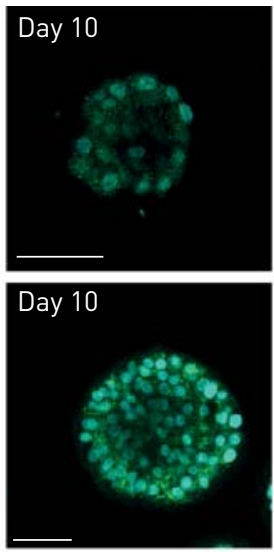

Overlay

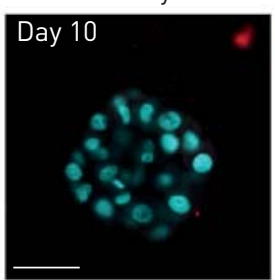

Day 10

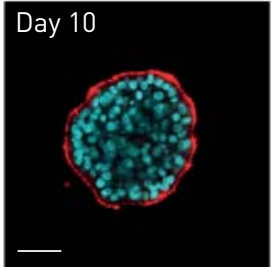

b)
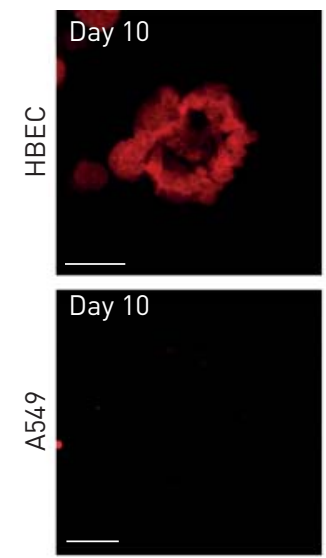

d) Anti-rabbit-546
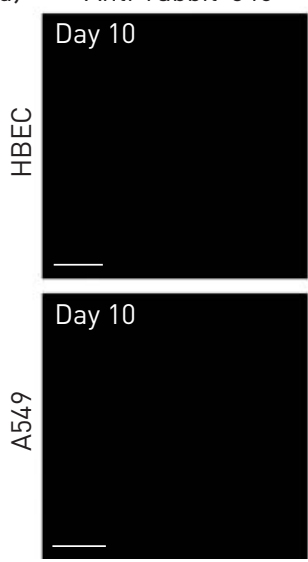

DAPI
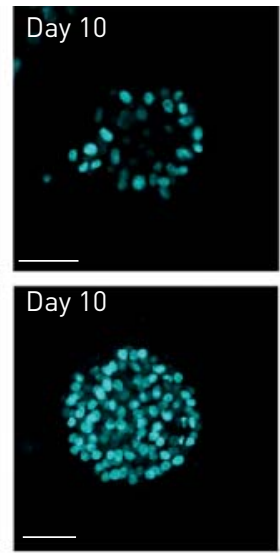

DAPI

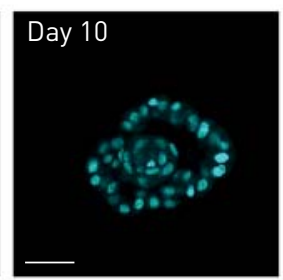

Day 10

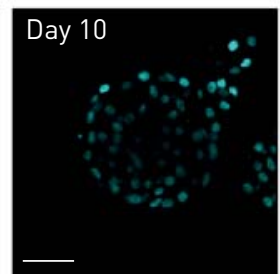

Overlay
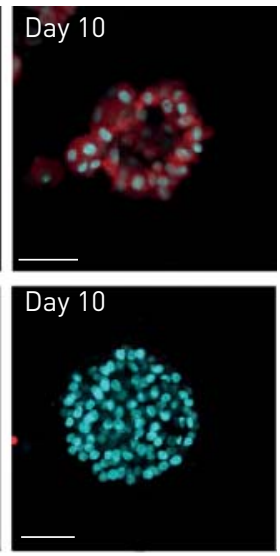

Overlay
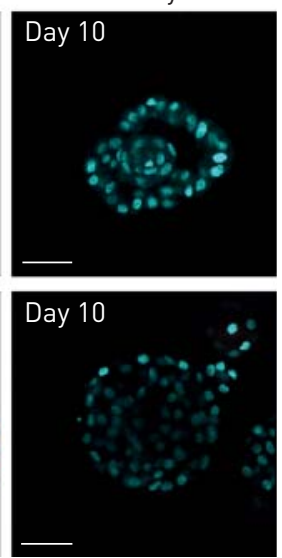

FIGURE 3 Immunofluoresence staining of human bronchial epithelial acini. Human bronchiol epithelial cells (HBECs) and cancer (A549) cells were grown on Matrigel and stained with a) mucin 5B antibody (MUC5B) (green), b) lysozyme antibody (AZGP1) (red) and c) $\beta$-tubulin IV antibody (red). d) Negative control

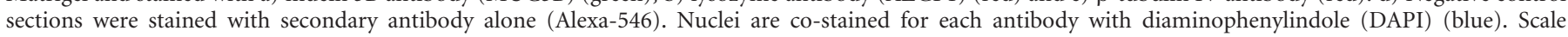
bars $=50 \mu \mathrm{m}$. Images were acquired by confocal microscopy Nikon Eclipse 2000-C1 (Nikon, Tokyo, Japan).

show that luminal apoptosis occurs during morphogenesis in HBEC and this differentiation capacity is lost for A549 cells. We further report the development and the morphological characterisation of the three-dimensional cell culture model system, utilising a panel of five lung NSCLC cell lines (online supplementary table S1). The adenocarcinoma and large cell undifferentiated carcinoma cells that we tested generated large spheroids lacking a hollow lumen (fig. 6a, three-dimensional brightfield) whereas the epidermoid carcinoma cell line formed stellate structures (fig. 6a, three-dimensional brightfield), characterised by the migration of chains cells. We also confirm for the other malignant cells (H23, H1838, H460 and Calu-1) (fig. 6), the p63 and DAPI staining pattern found in A549 cells (fig. 2b and 6), despite some distinct morphological pattern in two-dimensional-monolayer culture (fig. 6, twodimensional brightfield). The final mean diameter for HBECs is $\sim 82.81 \mu \mathrm{m}$, and for cancer cells A549, $\mathrm{H} 23, \mathrm{H} 1838, \mathrm{H} 460$ and Calu-1 is $87.04 \mu \mathrm{m}, 196.3 \mu \mathrm{m}, 167.2 \mu \mathrm{m}, 103.9 \mu \mathrm{m}$ and $103.1 \mu \mathrm{m}$, respectively (fig. 6b). The mean diameter of the acini in HBECs is consistent with previous reports of WU et al. [19]. In contrast, the different carcinoma cells form bigger acini, reaching $317 \mu \mathrm{m}$, all with a filled lumen. These two criteria (acini size and filled lumen) could therefore serve to easily distinguish normal cells from NSCLC lung cancer cells. To further confirm these results from cell lines with cells directly from lung biopsies, we grew primary HBECs from freshly isolated human biopsies of five different lung cancers. Figure 7 shows cells grown from the explants within 3-4 weeks in culture (fig. 7, lung explants column). Subculture in two-dimensions (fig. 7, two-dimensional brightfield) was verified using cytokeratins 14 and 18 staining (data not show) and growth in three-dimensional Matrigel. Within 10 days, we also observed the formation of sphere in the five samples tested (fig. 7, three-dimensionalbrightfield and DAPI panels). 
a)
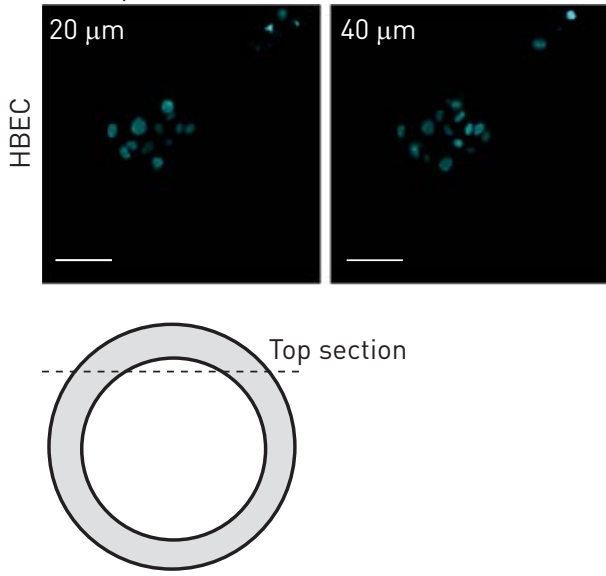

b)

Top section
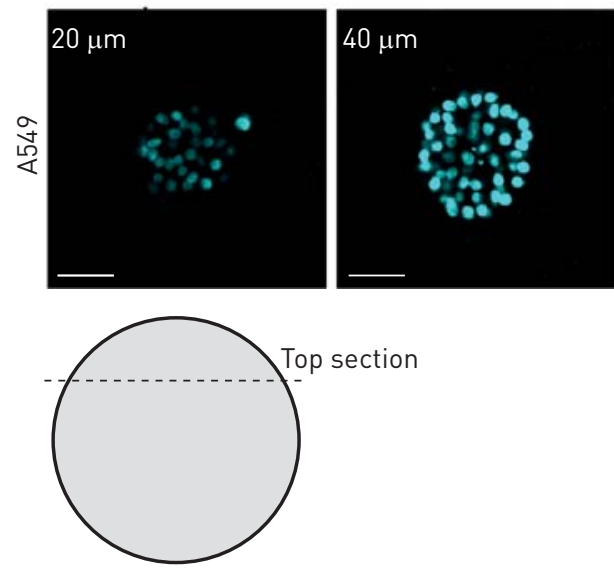

Lumen section
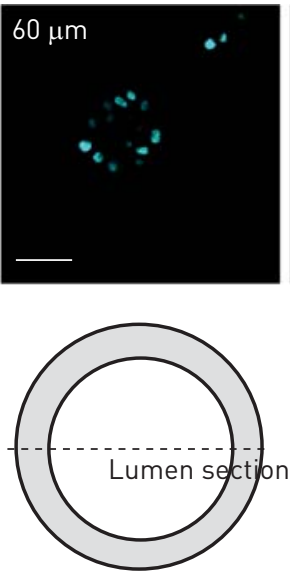

Middle section
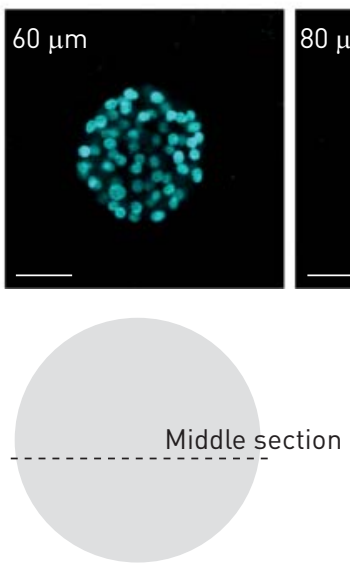

Bottom section
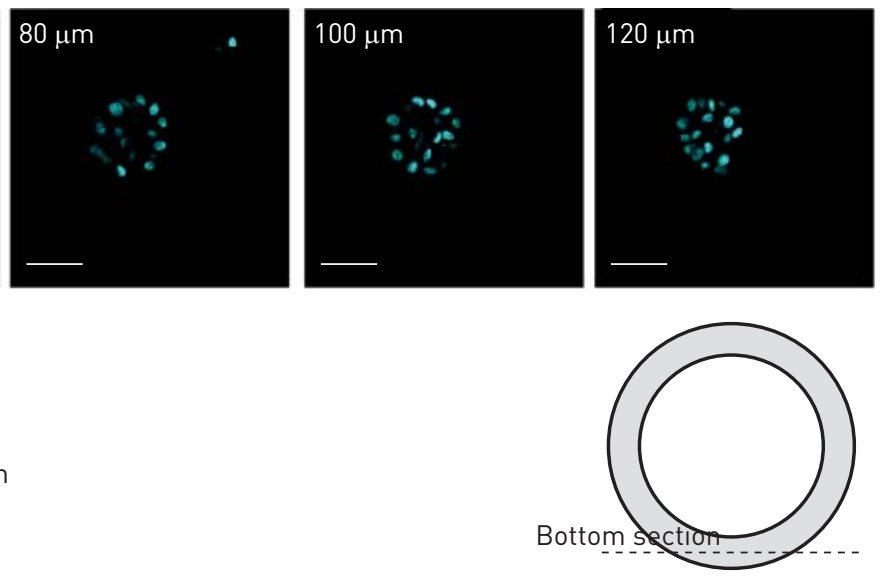

Bottom section
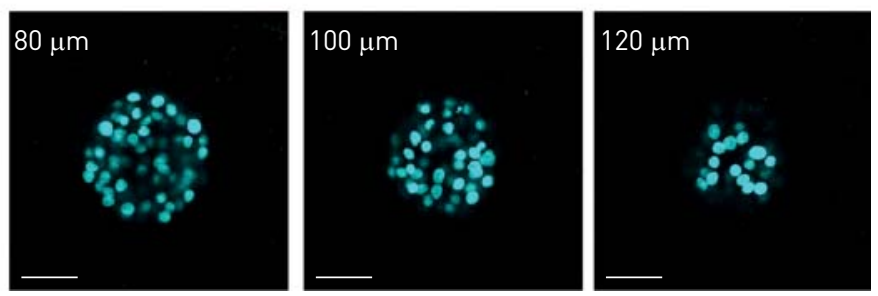

Bottom section

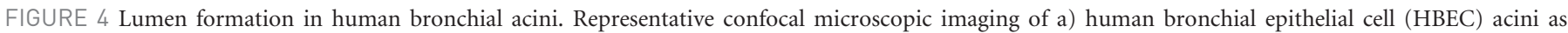
compared with b) A549 acini. Serial confocal z-stacks through day 13 acinus are shown. From the top to the bottom of acini grown in three dimensions, nuclei

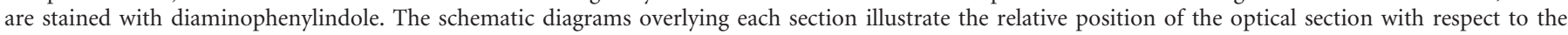
$z$-axis. The top and the bottom of the spherical structure appear as a sheet of cells because the optical section cuts across the surface contour of the sphere. In contrast, the middle section appears as a ring because the section cuts through the hollow lumen. Scale bars $=50 \mu \mathrm{m}$.

\section{Discussion}

The search for tumour-specific markers that could be used for the development of diagnostic and therapeutic strategies, especially for lung cancer, has been intense. Several differentiation markers, such as cytokeratin 19, have been studied for detection of lung adenocarcinoma. However, most of them are not specific for lung adenocarcinoma, but instead for epithelial cells or cancers of epithelial cell origin in general. Even for tissue-specific differentiation markers, such as the surfactant pulmonary-associated proteins A2, B and C and thyroid transcription factor-1, sensitivity as well as specificity is not high enough to use for molecular diagnosis $[32,33]$. Thus, it is absolutely necessary to search for more specific and highly sensitive markers that will be useful for molecular diagnosis of lung cancer.

Here, we have used a three-dimensional culture model for normal HBECs and NSCLC cells (online supplementary table S1). When cultured in extracellular matrix, HBECs re-acquire the ability to form acinilike structures presenting a hollow lumen. Presumably, the cells that die during lumen formation lack direct contact with the basement membrane, which renders them susceptible to anoikis, a form of programmed cell death that epithelial cells undergo on matrix detachment [34, 35]. Resemblance to acini in vivo was confirmed by the appearance of small connecting ductules between the spheres (fig. 1b), lumen formation in sectioned spheres (fig. 4a) and the expression of mucus and serous cell marker (fig. $3 \mathrm{a}$ and b). These structures recapitulate many of the characteristics of cell differentiation in the respiratory tract [36]. We can thus define markers for normal bronchial tissue in culture (online supplementary table S2). We also show, in contrast, the consistent inability of NSCLC cells to respond appropriately to this microenvironment. Other workers have used the matrix to distinguish between normal and human breast carcinoma cells [37]: studies revealed that human breast tumour cell lines did not form acini when grown in three-dimensional 

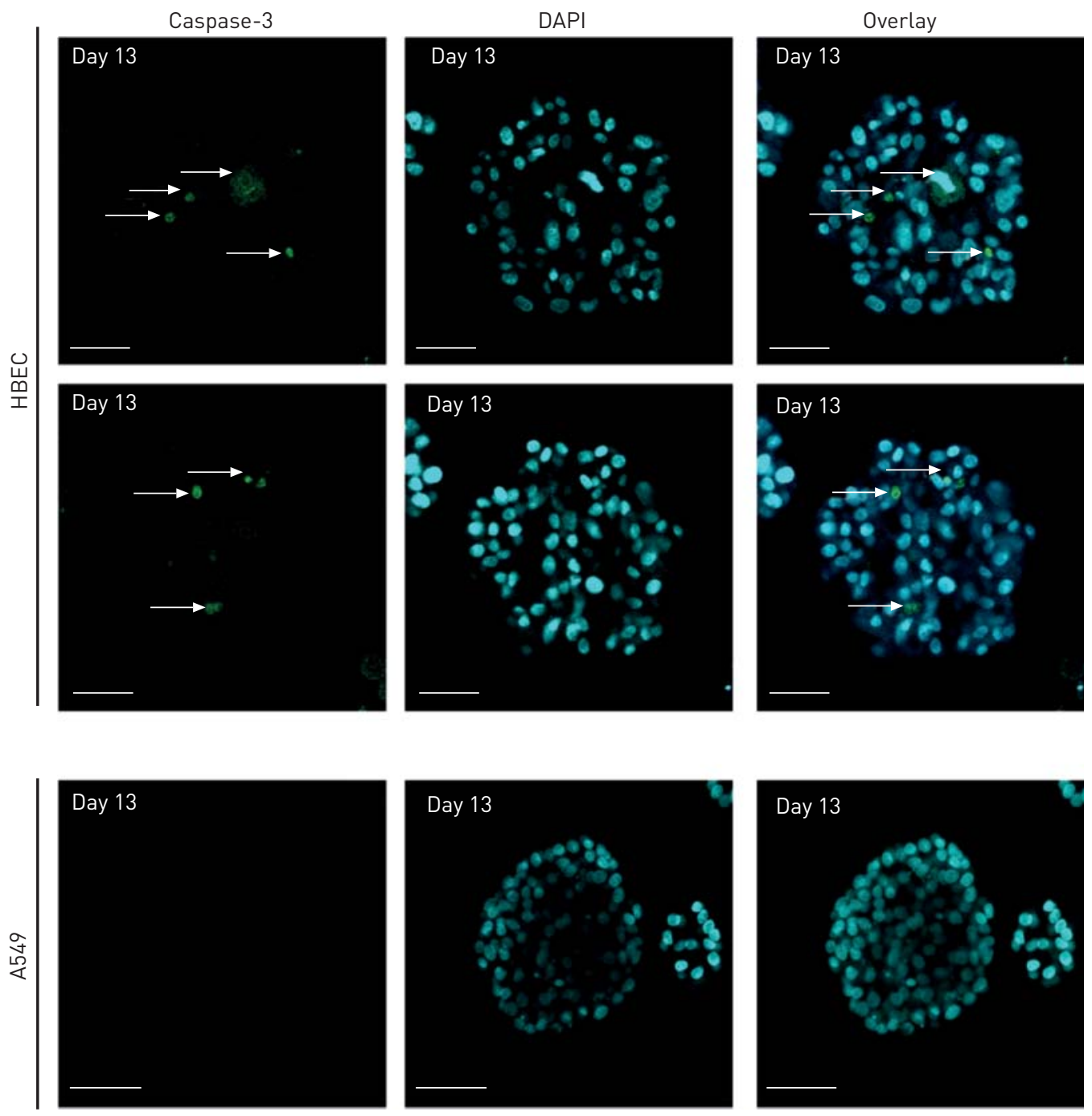

FIGURE 5 Apoptosis during lumen formation in human bronchial epithelial cells (HBECs) and not in A549 cancer cells. Cells were cultured on Matrigel for 13 days and were immunostained with antiserum to activated caspase 3 (green) to detect apoptosis and with diaminophenylindole (DAPI) to show nuclei (blue). Arrows represent caspase-positive cells. Scale bars $=50 \mu \mathrm{m}$.

culture; rather, they developed into nonpolarised clusters with limited differentiation. In our assay, all NSCLC lung epithelial cells (A549, H1838, H460, H23 and Calu-1) and cells freshly isolated from lung biopsies that grow in three-dimensions can be readily distinguished from normal HBECs (figs 6 and 7). In conclusion, we have shown that three-dimensional acini can now be readily established from the majority of clinical tumour-containing NSCLC specimens tested.

Our analysis revealed that lung cancer and lung normal epithelial cells were significantly different in threedimensional culture, suggesting that genes are being up- or downregulated specifically and commonly in lung cancer cells in comparison with lung normal epithelial cells. Subsequently, five markers were assessed as being differentially regulated in cancer cells in comparison with lung normal epithelial cells and, thus, as possible lung cancer-specific tumour markers (online supplementary table S2). To date, several genes have been used for the detection of lung adenocarcinoma cells, such as cytokine 19 and carcinoembryonic antigen $[38,39]$. However, both the sensitivity and specificity of these markers for detection of lung adenocarcinoma cells were not high enough to use as a single molecular marker in clinics. Thus, it will be important in future to further evaluate the specificity and sensitivity for the combined use of the five markers used in this study with several known markers for lung cancer cell detection. In figures 2 and 3, HBECs differentiated on Matrigel into polarised acinar cells expressed $\beta$-catenin (a cell junction marker), p63 (a basal cell marker), MUC5B (a marker of mucus cells and secretion), AZGP1 (a marker of serous cells and luminal secretion) and did not express $\beta$-tubulin IV (a marker for cilia). A549 cells expressed $\beta$-catenin 

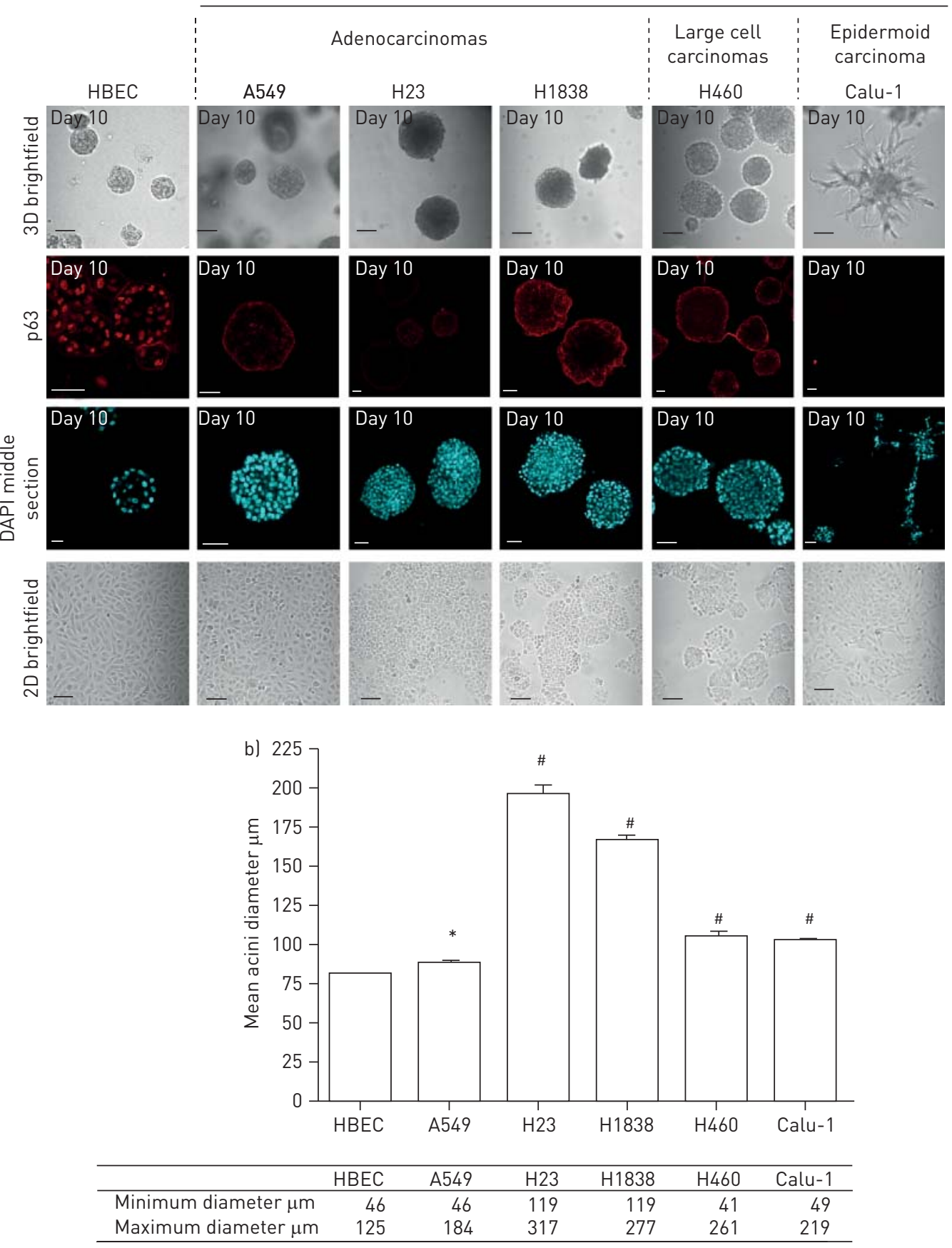

FIGURE 6 Inhibition of luminal apoptosis in epithelial lung cancer cells. a) 10-day-old structures of the indicated cell types (human bronchial epithelial cell (HBEC), A549, H23, H1838, H460 and Calu-1) were stained with diaminophenylindole (DAPI) to show nuclei (blue) and p63 (red). Representative confocal cross-sections through the middle of each structure (lumen sections) are shown (DAPI, middle panels). Phase contrast of cells grown in threedimensions (3D brightfield) and in two-dimensions (2D brightfield) are shown. Scale bars $=50 \mu \mathrm{m}$. b) Comparison of acini diameter in HBECs as compared with nonsmall cell lung cancer cells. Cells were grown on Matrigel for 13 days. Confocal images of acini were taken and their diameter measured by EZ-C1 3.9 Nikon software (Nikon, Tokyo, Japan). The maximum and minimum acini size $( \pm$ SEM $)$ is reported for each cell line. Statistical analyses were performed with a two-tailed t-test. A p-value $<0.05$ was regarded as statistically significant. ${ }^{\#}: \mathrm{p}<0.0001{ }^{\star}{ }^{\star}: \mathrm{p}<0.05$.

(fig. 2a), p63 (fig. 2b) and MUC5B (fig. 3a), but the localisation was different compared with HBECs (figs $2 \mathrm{a}$ and b, and 3a). In contrast, A549 cells compared with HBECs did not express AZGP1 (fig. 3b) or p63 (fig. 2b), but expressed $\beta$-tubulin IV (fig. 3c). The influence of p63 in the formation of acini has been 
HBEC_NSCLC \#002 Squamous cell carcinoma (grade 2)

HBEC_NSCLC \#004 Adenocarcinoma (grade 2)

HBEC_NSCLC \#007 Adenocarcinoma Igrade 2)

HBEC_NSCLC \#005 Adenocarcinoma (grade 2)

HBEC_NSCLC \#003 Adenocarcinoma (grade 3)
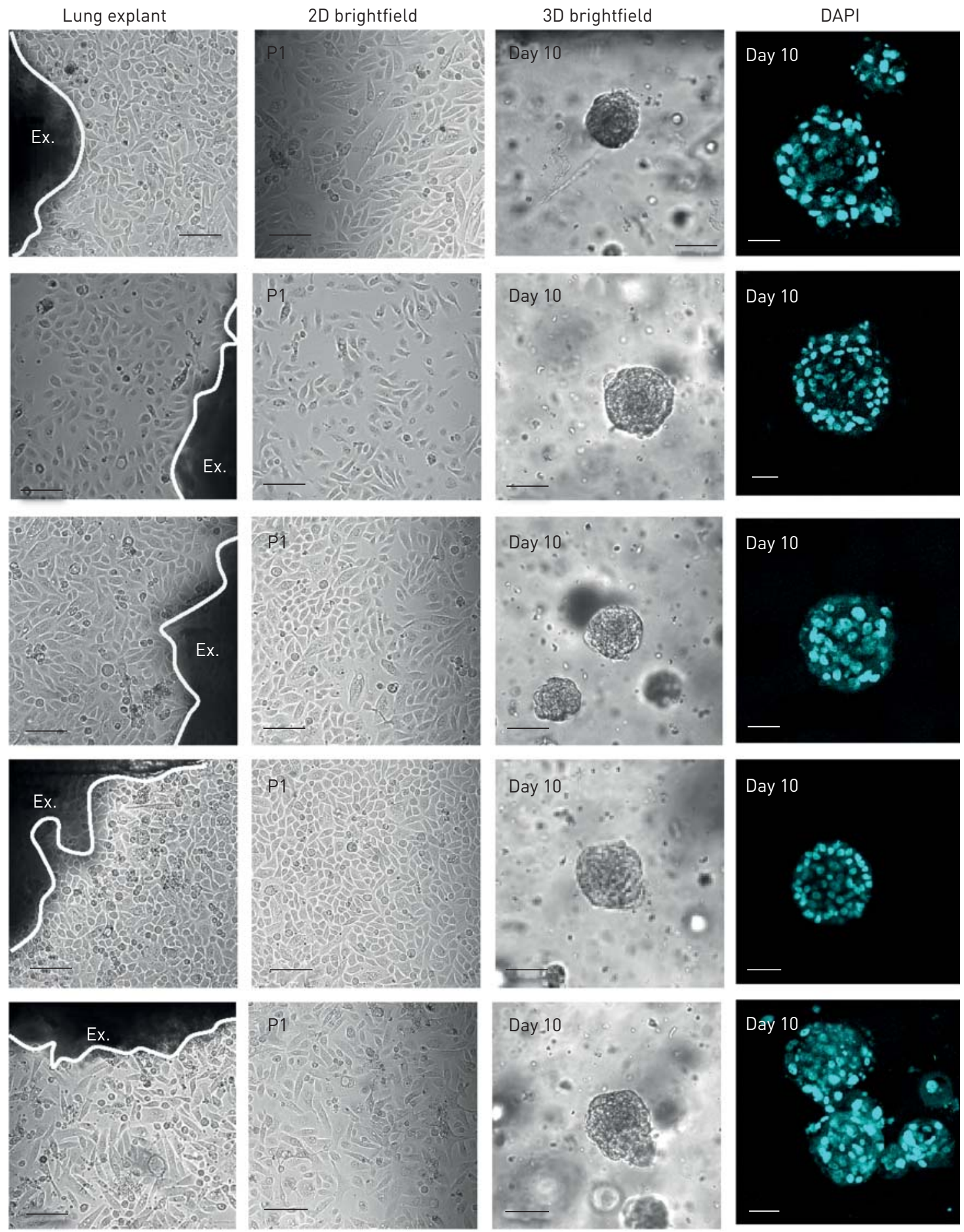

FIGURE 7 Three-dimensional (3D) acini formation with freshly isolated cells from lung biopsy of nonsmall cell lung cancer (NSCLC) patients. Primary human bronchial epithelial cells (HBECs) freshly isolated from human biopsies of five lung cancer growth from the explants (lung explants, far left column). Subculture in two-dimensions was established (2D brightfield) before growing cells in 3D Matrigel as spheres (3D brightfield). Representative confocal cross-sections through the middle of each structure (lumen sections) are shown (diaminophenylindole (DAPI) panels). Scale bars $=50 \mu \mathrm{m}$. Confocal images of acini were taken and their diameter was measured by EZ-C1 3.9 Nikon software (Nikon, Yokyo, Japan).

reported by SENOO et al. [40], where they have shown, using p63-depleted breast epithelial cells, that p63deficient cells yielded spheres, but fewer that one seventeenth the number seen in the wild-type. They propose that the decrease in the three-dimensional growth of the p63-null clones suggested a role for p63 in either the rate of proliferation or the overall proliferative potential of the epithelial cells rather than epithelial differentiation. 
In summary, the difference observed between normal and NSCLC cell lines (online supplementary table S1), both in the kinetics of differentiation and in the appearance of apoptosis (fig. 5 and 6) during formation of the lumen, and by the expression of acinar markers, as well as the formation of biologically relevant structures directly from lung biopsies, show that this model will provide a powerful tool for investigating mechanisms important in lung cancer pathological pathways. Thus, our experiments have illustrated the contrast between normal and tumour cells in three-dimensional culture, even though subtle phenotypic differences were evident when the same cells were grown as two-dimensional monolayers (fig. 6a, two-dimensional brightfield). It has been shown by proteomic approaches [41] that traditional monolayer cultures hardly recapitulate characteristics that result from the three-dimensional growth in tumours in vivo. Multicellular spheroid cultures are intermediates between monolayer cultures and tumours as they resemble the in vivo situation more closely with regard to cell proliferation, differentiation and cell environment, i.e. cell-cell contacts and different growth areas. The spheroids reflect the assembly of a tumour better than monolayer cultures and may therefore provide an advanced model system for in vivo tumours. For example, murine EMT6 mamma carcinoma cells exhibiting chemoresistance after treatment of the tumour-bearing mice recapitulated this resistant phenotype in multicellular spheroids but not in monolayer cultures [42]. Therefore, it is not surprising that the expression of AZGP1 and $\beta$-tubulin IV are different in two-dimensional (online supplementary fig. S2b and c) versus three-dimensional (online supplementary fig. S3b and c). Using a three-dimensional model enhanced our understanding of cancer biology in the formation and maintenance of a hollow lumen and its disruption in cancer cells. Implementation of this physiologically relevant model, illustrated here in the context of human lung epithelial tissues, enabled the study of intrinsic cell regulation pathways and also provided compelling evidence for the role of the stromal compartment in directing epithelial cell function and dysfunction. Thus, the utility of cancer cell acini grown in three-dimensional culture as an experimental tool is due largely to the ability to analyse the spatial and temporal aspects of key biological processes (e.g. proliferation and apoptosis), and signal transduction events during morphogenesis. The disruption of an intact glandular structure, including loss of apico-basal polarity and filling of the luminal space, is a hallmark of epithelial cancer, even at its earliest stages, yet very little is known about the mechanisms that elicit these changes during lung carcinoma formation. The morphogenesis of epithelial lung cancer acini in three-dimensional basement membrane culture provides a biologically rich system and the appropriate structural context in which to assay the effect of oncogenes on bronchial architecture. Notably, studies via the overexpression or silencing of specific genes in three-dimensional cultured cells broach the exciting possibility of employing gain/loss of function approaches in lung epithelial cells and, hence, facilitate the analysis of tumour suppressors on bronchial architecture.

The three-dimensional culture of HBECs in the matrix results in the formation of polarised, growtharrested acini-like spheroids that recapitulate several aspects of bronchial architecture in vivo. In conclusion, the development of such an in vitro model should provide new opportunities to gain biological insight into the mechanisms that promote the disruption of bronchial structure during lung carcinoma progression and help to create a mechanistic framework in the search for new diagnostic markers and therapeutic targets in lung cancers. Thus, this study illustrates the power of a three-dimensional epithelial culture model from cell lines and fresh biopsy specimens as a tractable in vitro approach to modelling the early events in lung carcinoma formation.

\section{References}

Yokota J, Kohno T. Molecular footprints of human lung cancer progression. Cancer Sci 2004; 95: 197-204. Sekido Y, Fong KM, Minna JD. Molecular genetics of lung cancer. Annu Rev Med 2003; 54: 73-87.

Dutt A, Wong KK. Mouse models of lung cancer. Clin Cancer Res 2006; 12: 4396s-4402s.

Travis WD, Brambilla E, Van Schil P, et al. Paradigm shifts in lung cancer as defined in the new IASLC/ATS/ERS lung adenocarcinoma classification. Eur Respir J 2011; 38: 239-243.

Travis WD. Pathology of lung cancer. Clin Chest Med 2011; 32: 669-692.

6 Bissell MJ, Radisky DC, Rizki A, et al. The organizing principle: microenvironmental influences in the normal and malignant breast. Differentiation 2002; 70: 537-546.

7 Shaw KR, Wrobel CN, Brugge JS. Use of three-dimensional basement membrane cultures to model oncogeneinduced changes in mammary epithelial morphogenesis. J Mammary Gland Biol Neoplasia 2004; 9: 297-310.

8 Wang F, Weaver VM, Petersen OW, et al. Reciprocal interactions between $\beta 1$-integrin and epidermal growth factor receptor in three-dimensional basement membrane breast cultures: a different perspective in epithelial biology. Proc Natl Acad Sci USA 1998; 95: 14821-14826.

9 Hogan BL, Kolodziej PA. Organogenesis: molecular mechanisms of tubulogenesis. Nat Rev Genet 2002; 3: 513-523.

10 Debnath J, Brugge JS. Modelling glandular epithelial cancers in three-dimensional cultures. Nat Rev Cancer 2005; 5: 675-688.

11 Martin KJ, Patrick DR, Bissell MJ, et al. Prognostic breast cancer signature identified from 3D culture model accurately predicts clinical outcome across independent datasets. PLoS One 2008; 3: e2994.

12 Roig AI, Eskiocak U, Hight SK, et al. Immortalized epithelial cells derived from human colon biopsies express stem cell markers and differentiate in vitro. Gastroenterology 2012; 138: 1012-1021. 
Townley AK, Schmidt K, Hodgson L, et al. Epithelial organization and cyst lumen expansion require efficient Sec13-Sec31-driven secretion. J Cell Sci 2012; 125: 673-684.

14 Wescott MP, Rovira M, Reichert M, et al. Pancreatic ductal morphogenesis and the Pdxl homeodomain transcription factor. Mol Biol Cell 2009; 20: 4838-4844.

15 Sempere LF, Gunn JR, Korc M. A novel 3-dimensional culture system uncovers growth stimulatory actions by TGF $\beta$ in pancreatic cancer cells. Cancer Biol Ther 2011; 12: 198-207.

16 Wu YM, Tang J, Zhao P, et al. Morphological changes and molecular expressions of hepatocellular carcinoma cells in three-dimensional culture model. Exp Mol Pathol 2009; 87: 133-140.

17 Deterding RR, Shannon JM. Proliferation and differentiation of fetal rat pulmonary epithelium in the absence of mesenchyme. J Clin Invest 1995; 95: 2963-2972.

18 Eblaghie MC, Reedy M, Oliver T, et al. Evidence that autocrine signaling through Bmprla regulates the proliferation, survival and morphogenetic behavior of distal lung epithelial cells. Dev Biol 2006; 291: 67-82.

19 Wu X, Peters-Hall JR, Bose S, et al. Human bronchial epithelial cells differentiate to 3D glandular acini on basement membrane matrix. Am J Respir Cell Mol Biol 2011; 44: 914-921.

20 Franzdóttir SR, Axelsson IT, Arason AJ, et al. Airway branching morphogenesis in three dimensional culture. Respir Res 2010; 11: 162.

21 Härmä V, Virtanen J, Mäkelä R, et al. A comprehensive panel of three-dimensional models for studies of prostate cancer growth, invasion and drug responses. PLoS One 2010; 5: e10431.

22 Schmeichel KL, Bissell MJ. Modeling tissue-specific signaling and organ function in three dimensions. J Cell Sci 2003; 116: 2377-2388.

23 Sharma P, Dudus L, Nielsen PA, et al. MUC5B and MUC7 are differentially expressed in mucous and serous cells of submucosal glands in human bronchial airways. Am J Respir Cell Mol Biol 1998; 19: 30-37.

24 Sommerhoff CP, Finkbeiner WE. Human tracheobronchial submucosal gland cells in culture. Am J Respir Cell Mol Biol 1990; 2: 41-50.

25 Blatchford DR, Quarrie LH, Tonner E, et al. Influence of microenvironment on mammary epithelial cell survival in primary culture. J Cell Physiol 1999; 181: 304-311.

26 Coucouvanis E, Martin GR. Signals for death and survival: a two-step mechanism for cavitation in the vertebrate embryo. Cell 1995; 83: 279-287.

27 Hoffman MP, Kibbey MC, Letterio JJ, et al. Role of laminin-1 and TGF-beta 3 in acinar differentiation of a human submandibular gland cell line (HSG). J Cell Sci 1996; 109: 2013-2021.

28 Huang J, Hardy JD, Sun Y, et al. Essential role of biliary glycoprotein (CD66a) in morphogenesis of the human mammary epithelial cell line MCF10F. J Cell Sci 1999; 112: 4193-4205.

29 Burstein HJ, Polyak K, Wong JS, et al. Ductal carcinoma in situ of the breast. N Engl J Med 2004; 350: $1430-1441$.

30 Lin HH, Yang TP, Jiang ST, et al. Bcl-2 overexpression prevents apoptosis-induced Madin-Darby canine kidney simple epithelial cyst formation. Kidney Int 1999; 55: 168-178.

31 Debnath J, Mills KR, Collins NL, et al. The role of apoptosis in creating and maintaining luminal space within normal and oncogene-expressing mammary acini. Cell 2002; 111: 29-40.

32 Chhieng DC, Cangiarella JF, Zakowski MF, et al. Use of thyroid transcription factor 1, PE-10, and cytokeratins 7 and 20 in discriminating between primary lung carcinomas and metastatic lesions in fine-needle aspiration biopsy specimens. Cancer 2001; 93: 330-336.

33 Zamecnik J, Kodet R. Value of thyroid transcription factor-1 and surfactant apoprotein A in the differential diagnosis of pulmonary carcinomas: a study of 109 cases. Virchows Arch 2002; 440: 353-361.

34 Boudreau N, Sympson CJ, Werb Z, et al. Suppression of ICE and apoptosis in mammary epithelial cells by extracellular matrix. Science 1995; 267: 891-893.

35 Frisch SM, Vuori K, Ruoslahti E, et al. Control of adhesion-dependent cell survival by focal adhesion kinase. $J$ Cell Biol 1996; 134: 793-799.

36 Reid CJ, Gould S, Harris A. Developmental expression of mucin genes in the human respiratory tract. Am J Respir Cell Mol Biol 1997; 17: 592-598.

37 Petersen OW, Rønnov-Jessen L, Howlett AR, et al. Interaction with basement membrane serves to rapidly distinguish growth and differentiation pattern of normal and malignant human breast epithelial cells. Proc Natl Acad Sci USA 1992; 89: 9064-9068.

38 Matsunaga H, Hangai N, Aso Y, et al. Application of differential display to identify genes for lung cancer detection in peripheral blood. Int J Cancer 2002; 100: 592-599.

39 Mitas M, Hoover L, Silvestri G, et al. Lunx is a superior molecular marker for detection of non-small cell lung cancer in peripheral blood. J Mol Diagn 2003; 5: 237-242.

40 Senoo M, Pinto F, Crum CP, et al. p63 is essential for the proliferative potential of stem cells in stratified epithelia. Cell 2007; 129: 523-536.

41 Gaedtke L, Thoenes L, Culmsee C, et al. Proteomic analysis reveals differences in protein expression in spheroid versus monolayer cultures of low-passage colon carcinoma cells. J Proteome Res 2007; 6: 4111-4118.

42 Kobayashi H, Man S, Graham CH, et al. Acquired multicellular-mediated resistance to alkylating agents in cancer. Proc Natl Acad Sci USA 1993; 90: 3294-3298. 